\title{
Journal of Artificial Organs 2018: the year in review
}

\author{
Journal of Artificial Organs Editorial Committee
}

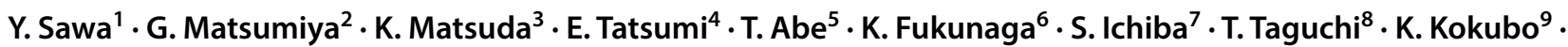 \\ T. Masuzawa ${ }^{10} \cdot$ A. Myoui ${ }^{11} \cdot$ M. Nishimura ${ }^{12} \cdot$ T. Nishimura ${ }^{13} \cdot$ T. Nishinaka ${ }^{14} \cdot$ E. Okamoto $^{15} \cdot$ S. Tokunaga ${ }^{16}$. \\ T. Tomo ${ }^{17} \cdot$ T. Tsukiya $^{18} \cdot$ Y. Yagi $^{19} \cdot$ T. Yamaoka ${ }^{20}$
}

Received: 29 January 2019 / Published online: 23 February 2019

(c) The Japanese Society for Artificial Organs 2019

\section{Introduction}

Members of the Editorial Committee of the Journal of Artificial Organs (JAO) are pleased to introduce to colleagues worldwide through the publication of JAO a broad spectrum of important new achievements in the field of artificial organs. We believe that JAO has a very high potential for promoting interest in the field of artificial organs. The impact factor announced in the Journal Citation Reports for 2017 was 1.224 . We are proud of this impact factor, which will certainly enhance international interest in the journal.

Y. Sawa

sawa@surg1.med.osaka-u.ac.jp

1 Division of Cardiovascular Surgery, Department of Surgery, Osaka University Graduate School of Medicine, Osaka, Japan

2 Department of Cardiovascular Surgery, Chiba University Graduate School of Medicine, Chiba, Japan

3 Emergency and Critical Care Medicine, University of Yamanashi Hospital, Yamanashi, Japan

4 Department of Artificial Organs, National Cerebral and Cardiovascular Center Research Institute, Osaka, Japan

5 Department of Urology, Iwate Medical University School of Medicine, Iwate, Japan

6 Faculty of Health Sciences, Kyorin University, Tokyo, Japan

7 Department of Surgical Intensive Care Medicine, Nippon Medical School Hospital, Tokyo, Japan

8 Biomaterial Unit, National Institute of Material Science, Ibaraki, Japan

9 Department of Medical Engineering and Technology, Kitasato University School of Allied Health Science, Kanagawa, Japan

10 Department of Mechanical Engineering, Ibaraki University, Ibaraki, Japan
From the beginning with Volume 1 in 1998 to the last issue (Volume 21) in 2018, we have received submissions from 40 countries, and we have accepted a total of 1078 papers for publication through the peer review process. In volume 21, we published 65 articles, amounting to 490 pages in total, including 42 original articles, 3 review articles, 13 case reports, and 6 brief communications. These papers were related to the many aspects of basic research, development, and clinical application of artificial organs, covering a variety of subfields. The yearly acceptance rate

11 Medical Center for Translational Research, Osaka University Hospital, Osaka, Japan

12 Division of Organ Regeneration Surgery, Tottori University Faculty of Medicine, Tottori, Japan

13 Department of Therapeutic Strategy for Heart Failure, The University of Tokyo, Tokyo, Japan

14 Department of Cardiovascular Surgery, Tokyo Women's Medical University, Tokyo, Japan

15 Department of Human Science and Informatics, School of Bioscience and Engineering, Tokai University, Sapporo, Japan

16 The Department of Cardiovascular Surgery, JCHO Kyushu Hospital, Fukuoka, Japan

17 Second Department of Internal Medicine, Faculty of Medicine, Oita University, Oita, Japan

18 Department of Artificial Organs, National Cerebral and Cardiovascular Center Research Institute, Osaka, Japan

19 Department of Clinical Engineering, Kyoto Prefectural University of Medicine, Kyoto, Japan

20 Department of Biomedical Engineering, National Cerebral and Cardiovascular Center Research Institute, Osaka, Japan 
was $54.1 \%$ in 2018 . This review summarizes the content of some of the most intriguing papers of the last year.

During the last year, a total of 134 reviewers who were specialists in artificial organs and interdisciplinary fields helped our authors to improve their manuscripts through thoughtful reviews, critiques, critiques, and suggestions. We are very happy to present such excellent work in JAO. We would like to express our profound gratitude to all authors, reviewers, and members from all over the world, and express the hope that you will continue to support our journal.

\section{Artificial heart (basic)}

Kishimoto et al. [1] developed a novel temporary LVAS for bridge to decision, in which a disposable centrifugal pump with a hydrodynamically levitated bearing was employed. They investigated the 30-day performance and hemocompatibility of the LVAS using three adult goats. All goats survived with conditions a mean pump flow was $3.2 \pm 0.6 \mathrm{~L} /$ $\mathrm{min}$ at 3000-4500 rpm and no damage caused by mechanical contact was found on the bearing. Excess hemolysis occurred in one case because they increased a rotational speed though sucking happened at the inflow cannula tip. At necropsy, an inconsiderable macroscopic renal infarction was observed in two goats, but no thrombus was observed in all pumps. Their LVAS demonstrated the consistent and satisfactory hemodynamic performance and hemocompatibility in the goat model.

Khan et al. [2] developed a new axial-flow blood pump: the shrouded impeller pediatric axial-blood pump. The shrouded impeller blood pump has a unique structure; it has two flow paths, primary path through impeller blades inside the shroud, and secondary path passing through ceramic bearings outside the shroud. For minimization of the blood damage in the ceramic bearings, Teflon baffle rings were introduced to reduce the blood flow outside the shroud. They examined the hydrodynamic performance and the degree of hemolysis by the CFD analysis and an in vitro experiment. They achieved a flow rate of $0.5-3.5 \mathrm{~L} / \mathrm{min}$ against a pump head of 67-105 $\mathrm{mmHg}$ at a rotational speed of 12,000 rpm, and they obtained the average NIH of $0.00674 \mathrm{~g} / 100 \mathrm{~L}$.

Iizuka et al. [3] investigated the effect of aortic insufficiency (AI) progression on hemodynamics and myocardial oxygen metabolism using an animal AI model wearing EVAHEART. The degree of the AI was controlled by changing the degree of inflation of the IVC filter placed at aortic valve position, and the degree of the AI was defined as the flow rate of LVAD-LV recirculation. With the progress of the AI, LVP and LAP were significantly increased while diastolic AoP was decreased. In addition, myocardial oxygen consumption was decreased while the rate of change of myocardial oxygen extraction ratio was increased with the AI progression. The results showed that the AI progression made it difficult to reduce cardiac load and it worsened myocardial metabolism.

\section{Artificial heart (clinical)}

Toda et al. [4] investigated the role of percutaneous venoarterial extracorporeal membrane oxygenation (VAECMO) as bridge to LVAD. They retrospectively investigated 32 LVAD patients who required VA-ECMO and found that a preoperative LVDd $\leq 54 \mathrm{~mm}$ was a significant predictor of 90-day mortality after LVAD implantation. Furthermore, preoperative LVDd was positively correlated with postoperative right ventricular function. They concluded that preoperative LVDd is a useful predictor of mortality and right ventricular function after LVAD implantation in patients with VA-ECMO.

Iizuka et al. [5] examined the effect of the angle between the outflow graft and the aorta $(\mathrm{O}-\mathrm{A}$ angle) on the hemodynamics of aortic insufficiency (AI) under LVAD support in an acute animal experimental model. An LVAD was installed in seven calves, with the inflow cannula inserted from the LV apex and with the outflow graft sutured at the ascending aorta. The AI model was made using a temporary inferior vena cava filter inserted from the LV apex and placed at the aortic valve. Cardiac dysfunction was induced by continuous beta-blocker infusion. Hemodynamic values and the myocardial oxygen extraction rate (O2ER) were evaluated at three $\mathrm{O}-\mathrm{A}$ angles $\left(45^{\circ}, 90^{\circ}\right.$, and $135^{\circ}$ ) over three levels of AI (none, Sellers I-II AI, and Sellers III-IV AI). The recirculation rate, defined as the percentage of regurgitation flow to LVAD output was, significantly increased with a larger $\mathrm{O}-\mathrm{A}$ angle. Coronary artery flow was decreased at a larger $\mathrm{O}-\mathrm{A}$ angle. O2ER tended to increase with a larger $\mathrm{O}-\mathrm{A}$ angle. A larger $\mathrm{O}-\mathrm{A}$ angle can increase the recirculation due to $\mathrm{AI}$ and can be disadvantageous to LVAD-AI hemodynamics and myocardial oxygen metabolism.

Anjum et al. [6] examined the incidence of postoperative acute kidney injury (AKI) in axial-flow (HeartMate II; HM-II) and centrifugal-flow (HVAD) CF-LVAD recipients, as well as the effect of AKI on mortality. They concluded that there was no significant difference in AKI rates for the HM-II and HVAD recipients, whereas patients with AKI after LVAD implantation had significantly reduced survival compared to patients without AKI.

Yoshitake et al. [7] reported that bridge-to bridge using Nipro p-VAD is a reasonable strategy for treating patients with severe decompensated end-stage heart failure, based on the results in which there was no significant difference in the incidence of infectious events or stroke as well as the 1- and 2-year survival rates. 


\section{Cardiopulmonary bypass}

Kobayashi et al. [8] investigated reginal cerebral oximetry using near-infrared spectroscopy during cardiopulmonary bypass using two different divices. Regional cerebral oximetry is widely used to avoid poor cerebral perfusion during cardiac surgery, but no definitive criteria of lower safe limit have been established. The authors found that an appropriate device should be selected according to preoperative patient characteristics, and that factors influencing regional cerebral oxygen saturation values should be considered to ensure the correct interpretation of measured values.

Shirakawa et al. [9] constructed 3D models of six prolapsed mitral valves for surgical assessment, and evaluated how accurately the models could replicate individual valve dimensions, to determining the complex geometry of mitral valve prolapse. They concluded that the $3 \mathrm{D}$ valve models could replicate the actual mitral valve prolapses within acceptable dimensional differences, and are useful for better $3 \mathrm{D}$ valve creation and better surgical planning with reliable $3 \mathrm{D}$ valve models.

\section{Artificial lung/ECMO}

Detection of clot formation inside cardiopulmonary support devices in an early phase is crucial to achieve a safe longterm support. Lazzeri et al. [10] found a promising marker for predicting ECMO circuit exchange because of circuit clots during ECMO support. They compared 10 vvECMOsupported cases retrospectively, and found that soluble fibrin (SF) was the only independent predictor of ECMO circuit exchange.

Murphy et al. [11] reported that a standardized approach to early continuous renal replacement therapy (CRRT) during neonatal extracorporeal life support (ECLS) results in greater homogeneity of CRRT initiation times with improvements in fluid balance and outcomes. They retrospectively analyzed the data (2007-2015) obtained from neonates treated prior to and after a 2011 practice change: CRRT initiation within $48 \mathrm{~h}$ of ECLS. This practice change resulted in improved short-term clinical outcomes including decreased weight gain at time of CRRT initiation as well as a more rapid return to baseline weight with early CRRT initiation, although no changes were found in duration of ECLS, duration of invasive ventilation, or survival.

Patients with sepsis or with acute lung failure often require deep sedation to tolerate invasive ventilation or to reduce patient-ventilator asynchrony during Veno-venous extracorporeal membrane oxygenation (vv ECMO). Axel et al. [12] reported a retrospective analysis of patients receiving vv ECMO support with inhalative sedation. They showed that even with smaller tidal volume due to lung rest settings for severe acute lung failure, targeted sedation with volatile anesthetics is feasible and dose-response relationship appears to exist.

Veno-venous extracorporeal membrane oxygenation (vv ECMO) is increasingly used as rescue therapy in severe respiratory failure. Frederik et al. [13] reported their retrospective study investigating outcomes of patients transferred to a specialized ECMO center after prolonged ECMO therapy ( $>8$ days on vv ECMO). 11/12 patients could be successfully weaned from ECMO.

In 7 patients, ECMO could be terminated after at least partial lung recovery, in 4 patients after salvage lung transplant. No patient died or needed re-initiation of ECMO therapy at day 28. They concluded that weaning from vv ECMO was feasible even after prolonged ECMO courses. Patients may benefit from transfer to a specialized ECMO center.

\section{Biomaterials}

Munisso et al. [14] developed of a simple in situ porcine closed-circuit system for assessing blood contacting biomaterials to explore the interaction between several materials and blood. This system allowed large numbers of materials (up to 16) to be simultaneously evaluated using the fewest number of animals. They compared modified ePTFE by coating with several peptides and quantified the thrombogenicity of the surfaces by adherent platelets, in vitro clotting kinetics, and in situ thrombogenicity of the materials using the closed-circuit system, and clarified the most appropriate modification of ePTFE surfaces to improve hemocompatibility.

\section{Tissue engineering/regenerative medicine}

The only radical treatment for acute hepatic failure is liver transplantation. Prior to liver transplantation, systemic management to prevent complications is important, and main therapies are artificial liver support and medical treatment such as drug therapy. One of the purposes of artificial liver support is to remove albumin-binding toxins and causative toxins in hepatic encephalopathy.

Viggiano et al. [15] compared the effects of bilirubin adsorption, plasma exchange and MARS in acute liver failure. Regarding the removal of bilirubin, the three therapies were equivalent. Moreover, they have reported that bilirubin adsorption is considered the first-line approach from the viewpoint of the cost and duration of treatment and MARS remains a valuable option in case of kidney involvement.

\section{Artificial kidney/dialysis}

Kurihara et al. [16] evaluated a continuous hemofiltration model using porcine blood. 
They used trisodium citrate in addition to nafamostat mesilate as anticoagulants.

The lifetime of the hemofilter was evaluated using the transmembrane pressure and the pressure drop across the hemofilter at varying trisodium citrate concentrations. They compared the lifetime of hemodiafilters among under several conditions (blood containing 7 or $8 \mathrm{mM}$ of trisodium citrate and nafamostat mesylate).

They conclude that a CHF model using porcine blood can be established by adjusting the concentration of trisodium citrate added to the blood.

\section{Artificial liver, pancreas}

The only radical treatment for acute hepatic failure is liver transplantation. Prior to liver transplantation, systemic management to prevent complications is important, and main therapies are artificial liver support and medical treatment such as drug therapy. One of the purposes of artificial liver support is to remove albumin-binding toxins and causative toxins in hepatic encephalopathy.

Viggiano et al. [17] compared the effects of bilirubin adsorption, plasma exchange and MARS in acute liver failure. Regarding the removal of bilirubin, the three therapies were equivalent. Moreover, they have reported that bilirubin adsorption is considered the first-line approach from the viewpoint of the cost and duration of treatment and MARS remains a valuable option in case of kidney involvement.

\section{Artificial skin, muscle, bone/joint, neuron}

Birmingham hip resurfacing (BHR) system is one of the representative surface replacing hip arthroplasty systems that has been reported to have several advantages compared with conventional stemmed-type total hip arthroplasty system. However, little is known about its results in Asian population. Sugano et al. [18] retrospectively studied the long-term results of BHR in Japanese patients and reported a good clinical outcome.

A computed tomography (CT)-based navigation system is one of the effective support tools in total hip arthroplasty (THA). Nakagawa et al. [19] evaluated the clinical results of THA using two different versions of a navigation system. The newer version of the navigation system with superior positional accuracy resulted in more accurate implant placement than old version.

\section{Others}

Toya et al. [20] evaluated intraoperative local oxygen supply-demand balance by monitoring regional saturation of oxygen $\left(\mathrm{rSO}_{2}\right)$ using near-infrared spectroscopy (NIRS), and analyzed the correlation between cannula size and fluctuation range of rSO, to prevent lower limb ischemia in minimally invasive cardiac surgery (MICS). They demonstrated that decreasing $\mathrm{rSO}_{2}$ correlates with the ratio of the cannula diameter to the femoral artery diameter $(\mathrm{Cd} / \mathrm{FAd})$ index. Low body surface area, $\mathrm{Cd} / \mathrm{Fad}>0.65$ is considered as the risk factor for decline of $\mathrm{rSO}_{2}$ in cannulated limb in MICS.

Morito et al. [21] investigated the oxygen consumption during hypothermic and subnormothermic machine perfusions (MP) of porcine liver grafts after cardiac death. This paper led the very fruitful conclusions that oxygen consumption indicated relationship between the hepatic artery pressure and effluent enzymes in LDH during MP, and that high oxygen consumption under subnormothermic MP $\left(22^{\circ} \mathrm{C}\right)$ offered several advantages over hypothermic $\mathrm{MP}\left(8-10^{\circ} \mathrm{C}\right)$ for donated after cardiac death liver graft preservation.

\section{References}

1. Kishimoto S, Takewa Y, Tsukiya T, Mizuno T, Date K, Sumikura H, Fujii Y, Ohnuma K, Togo K, Katagiri N, Naito N, Kishimoto Y, Nakamura Y, Nishimura M, Tatsumi E. Novel temporary left ventricular assist system with hydrodynamically levitated bearing pump for bridge to decision: initial preclinical assessment in a goat model. J Artif Organs. 2018;1:23-30.

2. Khan TI, Zad HS, Lazoglu I, Yalcin O. Development of a novel shrouded impeller pediatric blood pump. J Artif Organs. 2018; 2:142-9.

3. Iizuka K, Nishinaka T, Naito N, Akiyama D, Takewa Y, Yamazaki $\mathrm{K}$, Tatsumi E. Left heart pressures can be the key to know the limitation of left ventricular assist device support against progression of aortic insufficiency. J Artif Organs. 2018;3:265-70.

4. Toda K, Fujita T, Seguchi O, Yanase M, Nakatani T. Role of percutaneous veno-arterial extracorporeal membrane oxygenation as bridge to left ventricular assist device. J Artif Organs. 2018;1:39-45.

5. Kanjanahattakij N, Horn B, Abdulhadi B, Wongjarupong N, Mezue K, Rattanawong P. Blood stream infection is associated with cerebrovascular accident in patients with left ventricular assist device: a systematic review and meta-analysis. J Artif Organs. 2018;3:271-7.

6. Anjum A, Kurihara C, Critsinelis A, Kawabori M, Sugiura T, Civitello AB, Etheridge WB, Delgado RM, Simpson L, George JK, Nair AP, Frazier OH, Morgan JA. Acute kidney injury after implantation of a left ventricular assist device: a comparison of axial-flow (HeartMate II) and centrifugal-flow (HeartWare HVAD) devices. J Artif Organs. 2018;3:285-92.

7. Yoshitake S, Kinoshita O, Nawata K, Hoshino Y, Itoda Y, Kimura M, Yamauchi H, Ono M. Single-center experience of the bridgeto-bridge strategy using the Nipro paracorporeal ventricular assist device. J Artif Organs. 2018;4:405-11.

8. Kobayashi K, Kitamura T, Kohira S, Torii S, Mishima T, Ohkubo H, Tanaka Y, Sasahara A, Fukunishi T, Ohtomo Y, Horikoshi R, Murai Y, Miyaji K. Cerebral oximetry for cardiac surgery: a preoperative comparison of device characteristics and pitfalls in interpretation. J Artif Organs. 2018;4:412-8.

9. Toya T, Fujita T, Fukushima S, Shimahara Y, Kume Y, Yamashita K, Matsumoto Y, Kawamoto N, Kobayashi J. Efficacy of regional saturation of oxygen monitor using near-infrared spectroscopy for 
lower limb ischemia during minimally invasive cardiac surgery. $\mathbf{J}$ Artif Organs. 2018;4:420-6.

10. Lazzeri C, Bonizzoli M, Cianchi G, Batacchi S, Chiostri M, Fulceri GE, Buoninsegni LT, Peris A. Bilirubin in the early course of venovenous extracorporeal membrane oxygenation support for refractory ARDS. J Artif Organs. 2018;1:61-7.

11. Murphy HJ, Cahill JB, Twombley KE, Annibale DJ, Kiger JR. Implementing a practice change: early initiation of continuous renal replacement therapy during neonatal extracorporeal life support standardizes care and improves short-term outcomes. J Artif Organs. 2018;1:76-85.

12. Rand A, Zahn PK, Schildhauer TA, Waydhas C, Hamsen U. Inhalative sedation with small tidal volumes under venovenous ECMO. J Artif Organs. 2018;2:201-5.

13. Seiler F, Trudzinski FC, Hörsch SI, Kamp A, Metz C, Flaig M, Alqudrah M, Wehrfritz H, Kredel M, Muellenbach RM, Haake $\mathrm{H}$, Bals R, Lepper PM. Weaning from prolonged veno-venous extracorporeal membrane oxygenation (ECMO) after transfer to a specialized center: a retrospective study. J Artif Organs. 2018;3:300-7.

14. Munisso MC, Mahara A, Yamaoka T. Design of in situ porcine closed-circuit system for assessing blood-contacting biomaterials. J Artif Organs. 2018;3:317-24.

15. Lee JY, Park JH, Cho D-W. Comparison of tracheal reconstruction with allograft, fresh xenograft and artificial trachea scaffold in a rabbit model. J Artif Organs. 2018;3:325-31.
16. Hamada C, Nakamoto H, Suzuki Y. Morphologic characteristics of macroscopic peritoneal finding in patients with peritoneal dialysis. J Artif Organs. 2018;1:102-9.

17. Viggiano D, de Pascale E, Marinelli G, Pluvio C. A comparison among three different apheretic techniques for treatment of hyperbilirubinemia. J Artif Organs. 2018;1:110-6.

18. Uemura K, Takao M, Hamada H, Sakai T, Ohzono K, Sugano N. Long-term results of Birmingham hip resurfacing arthroplasty in Asian patients. J Artif Organs. 2018;1:117-23.

19. Soriano-Heras E, Blaya-Haro F, Molino C, Agustín del Burgo JM. Rapid prototyping prosthetic hand acting by a low-cost shapememory-alloy actuator. J Artif Organs. 2018;2:238-46.

20. Shirakawa T, Yoshitatsu M, Koyama Y, Kurata A, Miyoshi T, Mizoguchi H, Masai T, Toda K, Sawa Y. To what extent can 3D model replicate dimensions of individual mitral valve prolapse? J Artif Organs. 2018;3:348-55.

21. Morito N, Obara H, Matsuno N, Enosawa S, Furukawa H. Oxygen consumption during hypothermic and subnormothermic machine perfusions of porcine liver grafts after cardiac death. $\mathbf{J}$ Artif Organs. 2018;4:450-7.

Publisher's Note Springer Nature remains neutral with regard to jurisdictional claims in published maps and institutional affiliations. 